\title{
FODO-supercell based compact ring design with tunable momentum compaction and optimized dynamic aperture
}

\author{
Yipeng Sun* \\ SLAC, Stanford, CA 94025, USA
}

\begin{abstract}
A storage ring with tunable momentum compaction has the advantage in achieving different RMS bunch length with similar RF capacity, which is potentially useful for many applications, such as linear collider damping ring and predamping ring where injected beam has a large energy spread and a large transverse emittance. A tunable bunch length also makes the commissioning and fine tuning easier in manipulating the single bunch instabilities. In this paper, a compact ring design based on a supercell is presented, which achieves a tunable momentum compaction while maintaining a large dynamic aperture.
\end{abstract}

\section{Overview}

Storage rings based on alternate strong focusing are widely used for many years in design of colliders and synchrotron radiation light sources, also for linear collider damping ring design in the near term. Several focusing configurations have been invented and some of them applied in real ring construction and operation, including seperatedfunction FODO cell, combined function FD-cell, double-bend achromat (DBA), triple-bend achromat (TBA) [1] and theoretical minimum emittance lattice (TME) [2]. In an electron storage ring an electron will lose energy due to synchrotron radiations in the bending elements such as dipole magnets or wiggler. Since synchrotron radiation energy loss is compensated by the RF cavities in the ring, there exists a damping effect on the synchrotron oscillation with the corresponding damping time. Further more, the synchrotron radiation energy loss is in the form of randomly emitted photons, and the random quantum excitations together with the previously mentioned synchrotron radiation damping effects result in the single-particle equilibrium energy spread and emittance.

As discussed above, the equilibrium transverse emittance is achieved when radiation damping is equal to quantum excitation. The equilibrium transverse emittance in terms of the radiation integrals is listed below.

$$
\epsilon=C_{q} \frac{\gamma^{2} I_{5}}{J_{u} I_{2}}
$$

where $C_{q}=3.83 \times 10^{-13} \mathrm{~m}, \gamma$ denotes relativistic factor, $I_{2}$ and $I_{5}$ radiation integrals listed below.

$$
\begin{gathered}
I_{2}\left[m^{-1}\right]=\oint\left(\frac{1}{\rho_{x}^{2}}+\frac{1}{\rho_{y}^{2}}\right) d s \\
I_{5}\left[m^{-1}\right]=\oint\left(\frac{H_{u}}{\rho^{3}}\right) d s
\end{gathered}
$$

where $H_{u}=\beta_{u} D_{u}^{\prime 2}+2 \alpha_{u} D_{u} D_{u}^{\prime}+\gamma_{u} D_{u}^{2}$ depends on the bending and focusing structure, $\rho_{x}$ denotes the bending curvature in horizontal plane, $\rho_{y}$ bending curvature in vertical plane.

Observed from the three formulae above, one notes that in order to get a smaller equilibrium emittance at a given energy, $\rho$ need to be large and $H_{u}$ need to be small. A large $\rho$ requirement translates into more (in number) and

\footnotetext{
*Electronic address: yisun@slac.stanford.edu
} 
longer dipole magnets, while a small $H_{u}$ push the focusing structure evolvement, such as DBA, TBA and TME. Another version of the equilibrium emittance in terms of the bending/focusing properties is shown below.

$$
\epsilon=\frac{F}{12 \sqrt{15}} C_{q} \gamma^{2} \frac{\theta^{3}}{J_{x}}
$$

where $\theta$ denotes bending angle in each dipole magnet, $F$ a dimentionless factor depending on the lattice structure of the arc cell. Studies show that the minimum emittance could be achieved for TME arc cell with a corresponding $F=1[2]$.

In general, requirement on achieving a low emittance means employment of weaker dipole (smaller dispersion) and stronger quadrupole (focusing). The betatron oscillation tune variation of off-momentum particles with respect to the on-momentum particle is characterized by the chromaticity $\Delta \nu=\xi(p) \Delta p / p_{0}$. The natural chromaticity from the linear optics is always negative, $\xi_{0}=-\frac{1}{4 \pi} \oint K \beta d s$. Sextupoles are pure second order magnetic elements and then can be placed at dispersive regions where offset is proportional to momentum deviation, and provide extra focusing for off-momentum particles. The contribution of a single sextupole magnet with length $l_{s}$ to the total chromaticity is shown below, which being located at a place with dispersion $D$ in the bending plane.

$$
\Delta \xi= \pm \frac{1}{4 \pi} D \beta \frac{B^{\prime \prime} l_{s}}{B \rho}
$$

where $\beta$ denotes the beta function at the sextupole location, $B^{\prime \prime}$ sextupole strength, $B \rho$ beam magnetic rigidity and + sign associated with the bending plane.

One observes that there is a large natural chromaticity associated with a strong focusing lattice (large $K$ ), at the mean time $\beta$ tends to be smaller and so does dispersion $D$. All these make the chromaticity correction sextupoles stronger which in turn decrease the dynamic aperture which is a measure of the maximum stable phase space amplitude in transverse planes. An adequate dynamic aperture is essential in accepting the injected beam which usually has large emittance and energy spread, also in achieving a longer life time when stored in the ring.

There are several different approaches to evaluate and study the mechanism of dynamic aperture. One such approach as the matrix formulae is developed by K. Brown and applied to design second order or even higher order achromat [3]. Here we briefly review the main conclusions which are described in the following. Any optics with $n$ (larger than one) identical cells gives a first order achromat if the betatron phase advance equals $2 \pi$ in both transverse planes (first order transport matrix equaling unity $I$ ). When the cell number $n$ does not equal three, the second order geometric aberrations are also canceled. The second point is, of all the second order chromatic aberrations only two are independent, which then can be corrected with two families of sextupoles in each transverse plane.

A general matrix notation for the transport of particles' coordinates is shown in the following formula, which has first order, second order and third order terms [3].

$$
x_{i}(1)=\sum_{j} R_{i j} x_{j}(0)+\sum_{j, k} T_{i j k} x_{j}(0) x_{k}(0)+\sum_{j, k, l} U_{i j k l} x_{j}(0) x_{k}(0) x_{l}(0)
$$

where second order matrix $T$ has entries of the quadratic terms in the expansion of the final coordinates as a function of the initial coordiantes, and third order matrix $U$ has entries of the cubic terms.

Let any particle with coordinates $\left(x, x^{\prime}, y, y^{\prime}, z, \delta_{p}\right)$ passing by a second order achromat, the final coordinate would be the same as the initial one, as $R$ is a unity matrix and all $T$ matrix elements equals zero (neglecting higher order effects above three). That points to a way of evaluate and improve the dynamic aperture in a storage ring for the ideal case, where one can simply design a ring optics which consists of second order achromats regarding both geometric and chromatic aberrations. W. Wan developed another approach with Lie algebra to design general achromat to arbitrary order, taking advantage of the midplane symmetry and using multipole magnets for each order (for example, octupoles for third order achromat) [4]. The details are not discussed here. 

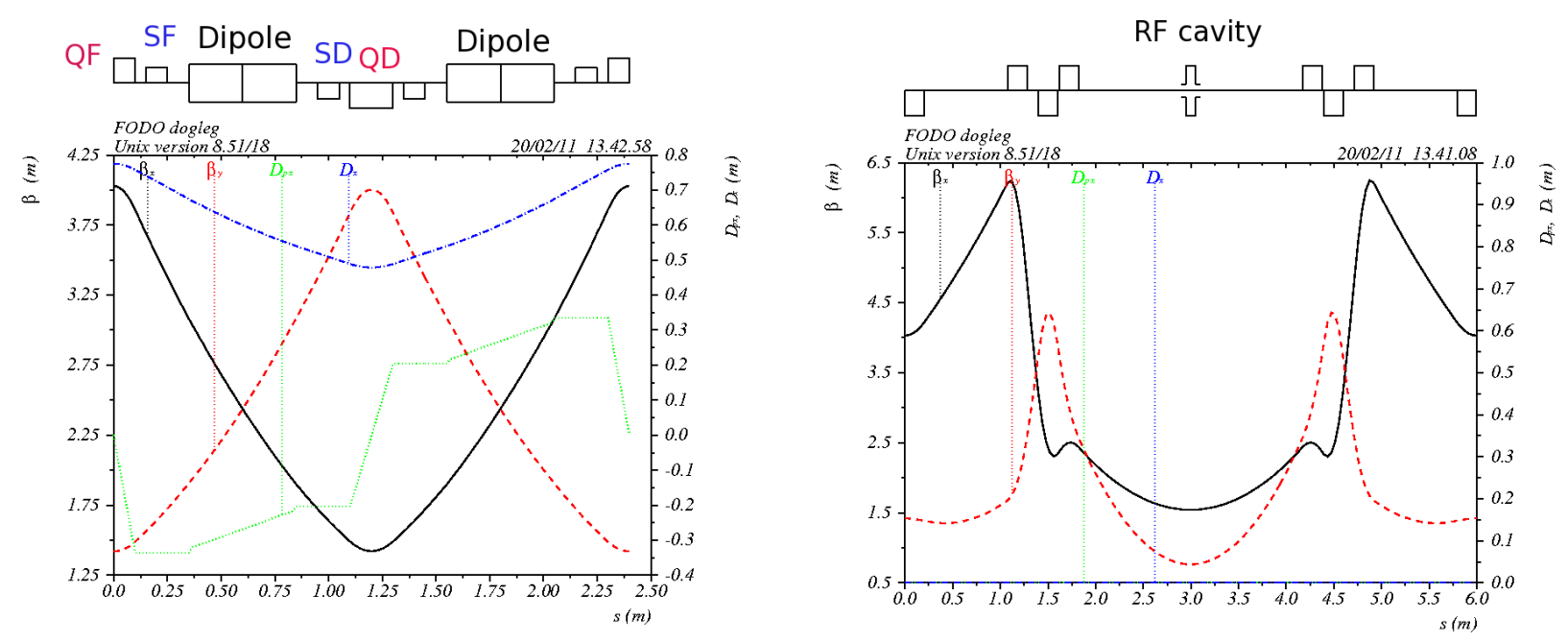

Figure 1: First order dispersion and beta functions. Left: one FODO cell; Right: one straight section cell which contains an RF cavity.

Recently Y. Cai has demonstrated that all driving terms up to fourth order resonance from chromaticity correction sextupoles can be canceled out within each arc achromat section, by derivations using Lie algebraic method [5]. An example lattice composed of TME arc cell is given, where one section consists of eight TME cell and the horizontal and vertical betatron phase advance is chosen to be $135 / 45$ degree in each cell.

In the following sections, a second order achromatic supercell is designed based on K. Brown's theory [3], where all geometric and chromatic aberrations vanish. Standard FODO cells are employed. The betatron phase advance of this supercell can be tuned in a wide range, which then can provide a flexible momentum compaction. Storage rings can be designed with its arc being constructed with several of such identical supercells and a decreased bending angle for each dipole magnet. This design supresses the need to add extra dispersion suppressors between the arc section and straight section.

\section{Single cell design}

First let us try to design a small compact ring with a circumference around $70 \mathrm{~m}$. The design goal of the arc cell is compact, simple and flexible. A standard FODO cell is picked as the basic unit, with a cell length roughly as $2.4 \mathrm{~m}$. The dipole length is chosen to be $0.5 \mathrm{~m}$ (just for this example), and the drift length is $0.25 \mathrm{~m}$ between each dipole and quadrupole magnet. A quadrupole length of $0.2 \mathrm{~m}$ and a sextupole length of $0.1 \mathrm{~m}$ are adopted, which can be made longer if necessary. There are two focusing sextupoles placed at both sides of one focusing quadrupole to correct the linear chromaticity (second order chromatic aberrations). For the defocusing quadrupole the arrangement is similar. This standard FODO cell maintains a midplane symmetry, as can be observed from its first order optics shown in Figure 1 (left). The betatron phase advance equals 60 degree in both transverse planes. For other possible ring designs, such as a synchrotron radiation light source with ultra low emittance, the dipole length can be further increased and the dispersion will be decreased. The phase advance can be kept the same by tuning the quadrupole strength.

The maximum and minimum $\beta$ function depends on the cell length and quadrupole strength (for a standard FODO cell), as shown below. 


$$
\beta \pm=\frac{2\left(1 \pm \frac{L_{\text {cell }}}{4} K_{1}\right)}{K_{1} \sqrt{1-\left(\frac{L_{c e l l}}{4} K_{1}\right)^{2}}}
$$

where $\beta \pm$ denotes the maximum and minimum beta functions, $L_{\text {cell }}$ the FODO cell length and $K_{1}$ the integrated quadrupole strength.

In Figure 1 (right) the first order optics of a straight section cell is shown, where midplane symmetry is again preserved. Four quadrupoles are used in each half, to match the beta function and tune the phase advance. The straight section can be composed of such cells and the FODO cells discussed above with the dipole magnets being replaced by drift space. The computer code MAD8 is used for optics matching [6].

\section{Supercell for three phase advance}

According to Brown's theory, a supercell which consists of more than four identical cells (such as the FODO cell discussed above) can make a second order achromat, given the betatron phase advance equaling a multiple of $2 \pi$ in both transverse planes. The second order geometric aberrations are canceled between these identical cells without sextupole assistance. Two group of sextupoles in each cell then correct second order chromatic aberrations and do not introduce new second order geometric aberrations. With this kind of configuration, the first order and second order transport matrix through one supercell are listed below. One observes that any particle's 6-D coordinates are reproduced up to second order.

$$
\begin{gathered}
\mathbf{R}=\mathbf{I} \\
T_{i j k}=0
\end{gathered}
$$

Given a fixed arrangement of the dipole magnets constrained by the ring geometry, one can adjust the quadrupoles to tune the dispersion function and the momentum compaction. A supercell which contains 12 standard FODO cells is chosen here to construct one achromat module. Three different horizontal betatron phase advance are considered, which are 30 degree, 60 degree and 90 degree, respectively. In all three cases, the total phase advance is a multiple of $2 \pi$, which ensures that the overall transport matrix is a unity matrix up to second order. There are 24 dipole magnets in each supercell.

Take a bending angle of 7.5 degree for each dipole magnet, the first order optics of such a supercell is shown in Figure 2, for three different phase advance, respectively. The total bending angle of this supercell is 180 degree, and its length is roughly $29 \mathrm{~m}$.

\section{Small ring design and dynamic aperture}

The geometry of the ring takes a racetrack shape. One supercell composes of a half arc section which is almost a half ring. For simplicity, here only two straight section cells as shown in Figure 1 (right) are used as the matching section between the two arcs. The key point is that the phase advance in each arc section is an integer, and the second order aberrations vanish inside each arc section. The straight section can then be used to accommodate other components, such as injection/extraction, RF cavities, damping wigglers, and insertion devices (undulators) for light sources. The overall betatron tune can be controlled by tuning the phase advance with quadrupole magnets in the straight sections. 

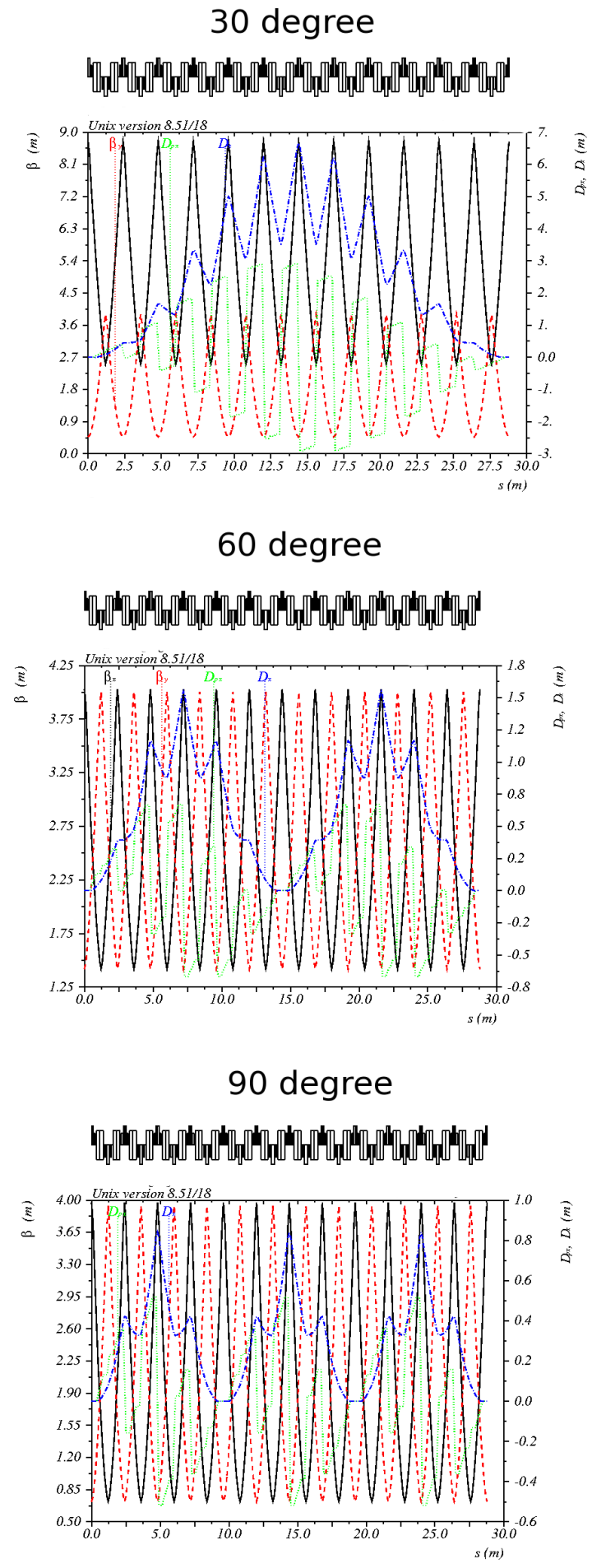

Figure 2: First order dispersion and beta functions. Top: 30 degree horizontal betatron phase advance in each FODO cell, a total phase advance of $2 \pi$; Middle: 60 degree horizontal betatron phase advance in each FODO cell, a total phase advance of

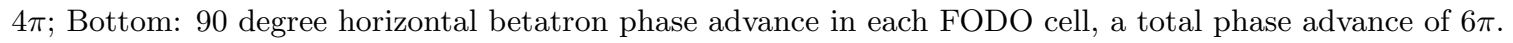




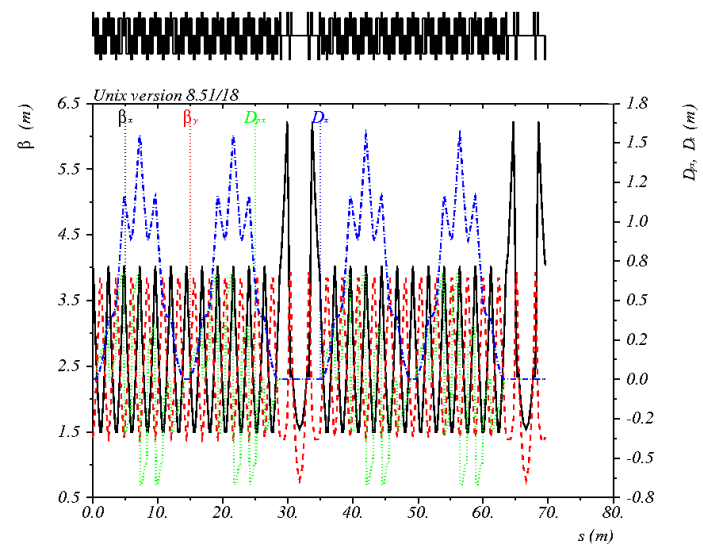

Figure 3: First order dispersion and beta functions of an example ring design. The betatron phase advance of one FODO cell equals 60 degree in both transverse planes.

As there are only drifts and quadrupoles in the straight section, there is no geometric aberrations induced. However, there are chromatic aberrations contributed from the straight section which adds on the natural chromaticity. The arc sextupoles then need to be tuned again to compensate the extra chromaticity.

One such a compact ring design is shown in Figure 3 with its first order optics, where one observes that there are two arc sections and two short straight sections. Each dipole magnet bends the beam for 7.5 degree in the horizontal plane. Dispersion function closes in each supercell and there are four periods of oscillations in all. After slightly tuning the arc sextupoles to cancel the chromaticity from the straights, a unity transport matrix is achieved up to second order, which is also confirmed numerically in MAD8 [6]. The circumference of the ring is roughly $70 \mathrm{~m}$.

As discussed above, in such a ring one can easily tune the phase advance of each FODO cell to be either 30 degree or 90 degree without moving any magnets, and achieve different momentum compaction and RMS bunch length for a given RF system. The straight section need to be slightly tuned to match the new TWISS parameters. One need to note that in all cases, the alpha function (derivative of beta function) is always zero at both ends of one supercell, which is essential in making it extendable. A comparison of different key parameters between these three operating options is listed in Table 1 below, for an electron beam at an energy of $1 \mathrm{GeV}$. The RF frequency is chosen to be $200 \mathrm{MHz}$.

Table I: Comparison of different key parameters between these three operating options (electron energy of $1 G e V$ ).

\begin{tabular}{lcccc}
\hline Parameter & unit & 30 degree & 60 degree & 90 degree \\
\hline Circumference & $m$ & 70 & 70 & 70 \\
Phase advance x/y (supercell) & $2 \pi$ & $1 / 4$ & $2 / 2$ & $3 / 3$ \\
Tune x/y/s & $2 \pi$ & $2.4 / 8.8 / 0.06$ & $4.3 / 4.7 / 0.03$ & $6.4 / 6.7 / 0.02$ \\
Natural chromaticity x/y & & $-2.5 /-6.1$ & $-4.9 /-5.6$ & $-8.5 /-8.6$ \\
RF voltage & $M V$ & 2 & 2 & 2 \\
Momentum compaction & $10^{-2}$ & 18 & 5 & 3 \\
Emittance & $\mu m \cdot r a d$ & 2 & 0.14 & 0.04 \\
Damping time & $m s$ & 60 & 24 & 19 \\
Energy spread & $10^{-3}$ & 0.38 & 0.42 & 0.43 \\
RMS bunch length & $m m$ & 15 & 9 & 6 \\
\hline
\end{tabular}

Dynamic aperture should ensure efficient acceptance of the injected beam with large emittance and energy spread. 

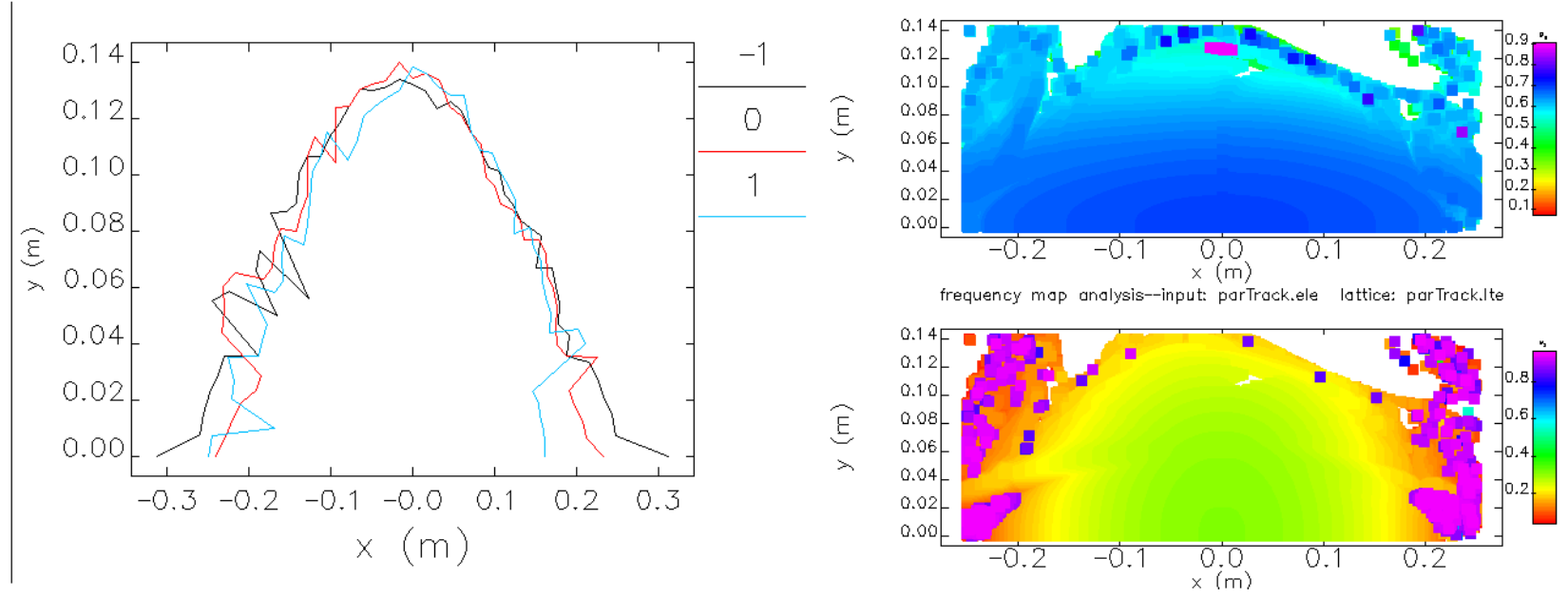

Figure 4: Left: dynamic aperture of on-momentum and off-momentum ( $\pm 1 \%)$ particles over 1000 turns of tracking; Right: tune footprint in the $\mathrm{x}-\mathrm{y}$ phase space over 1000 turns, horizontal tune (top) and vertical tune (bottom).

The natural chromaticity from the straight section need to be minimized which in turn requires weaker arc sextupoles. Usually dynamic aperture is determined by single particle numerical tracking simulation. For electron storage rings, synchrotron radiation damping plays an important role and the number of turns one need to track for dynamic aperture depends on damping time. In general one damping time is thousands of turns, and it is sufficient to evaluate the dynamic aperture for particles which can survive $10 \%-15 \%$ of damping time.

For this compact ring running at $2 \mathrm{GeV}$, one synchrotron oscillation period is roughly 70 turns, and one damping time is corresponding to $10^{4}$ turns. The full optics is then converted into the computer code Elegant [7], and the dynamic aperture is searched at 53 different angles in $\mathrm{x}-\mathrm{y}$ phase space for both on-momentum and off-momentum particles. For each angle, a single particle is launched for tracking with its initial coordinate setting at small amplitude to large amplitude. Momentum offset up to $\pm 1 \%$ is considered, and the 1000 turns dynamic aperture is shown in Figure 4 (left). One observes that in the horizontal direction a maximum value of $0.2 \mathrm{~m}$ is achieved, and it is $0.14 \mathrm{~m}$ in the vertical direction. That translates into $10^{4}$ times of equilibrium RMS beam size $\left(10^{4} \sigma\right)$ at $2 \mathrm{GeV}$. There is no obvious deviation for off-momentum particles.

The tune footprint is also investigated for 1024 turns, with the results shown in Figure 4 (right). One observes that there is no amplitude related detuning, with an initial transverse offset up to $0.15 \mathrm{~m}$ which is usually much larger than the physical aperture. This small ring design has a relatively large emittance, which is $0.04 \mu \mathrm{m} \cdot \mathrm{rad}$ at a beam energy of $1 \mathrm{GeV}$, for 90 degree case. Due to this limitation, it may be used for a pre-damping ring or a booster ring.

\section{Conclusion and discussion}

A compact ring design is presented in this paper which has a tunable momentum compaction and large dynamic aperture, based on K. Brown's achromat theory. One supercell consists of 12 identical FODO cells and it is a basic module to construct a ring. As an example two such supercells are employed to construct a small ring which has a pontential suitable for a pre-damping ring or a booster ring. The transport matrix through the arc section is always a unity matrix up to second order. The overall betatron tune is controlled in the straight sections. The dipole length and number can be largely increased to convert this compact ring into a low emittance ring with a circumference in the hundreds or kilo meter range. As an example, one can increase the dipole length from $0.5 \mathrm{~m}$ to $2.7 \mathrm{~m}$, and decrease the bending angle from 7.5 degree to 0.75 degree. Twenty such new supercells could be employed to construct a low emittance storage ring, with a geometric emittance of $0.5 \mathrm{~nm} \cdot \mathrm{rad}$ at a beam energy of $5 \mathrm{GeV}$. Compared with similar 
low emittance rings based on TME cells for ILC damping ring, the cost is more number of magnets and longer length of arc sections $(2 \mathrm{~km})$. Reducing the beam energy to $2 \mathrm{GeV}$ makes $1 \mathrm{~km}$ arc section possible, while it still provides a geometric emittance of $0.7 \mathrm{~nm} \cdot \mathrm{rad}$. A 120 degree FODO cell option is being investigated which may need less magnets and shorter arc length.

\section{Acknowledgement}

The authors would like to thank M. Woodley, W. Wan, Y. Cai for helpful discussions, also C. Adolphsen for his support.

This work was supported by the DOE under Contract DE-AC02-76SF00515.

\section{References}

[1] A. Chao and M. Tigner, Handbook of Accelerator Physics and Engineering, World Scientific, Singapore, page 76-81 (1998).

[2] L. Teng, Fermilab Report No. TM-1269 (1984); P. Emma and T. Raubenheimer, Phys. Rev. ST Accel. Beams 4, 021001 (2001).

[3] K. Brown, IEEE Trans. Nucl. Sci. NS-26 (3), 3490 (1979).

[4] W. Wan and M. Berz, Phys. Rev. E 54, 2870-2883 (1996).

[5] Y. Cai, to be published on Nuclear Instruments and Methods in Physics Research Section A, doi:10.1016/j.nima.2010.12.002 (2011).

[6] H. Grote, F.C. Iselin, The MAD Program (Methodical Accelerator Design) Version 8.15, CERN/SL/90-13 (AP), 1990.

[7] M. Borland, 'elegant: A Flexible SDDS-Compliant Code for Accelerator Simulation', Advanced Photon Source LS-287, September 2000. 